\title{
Coordination of Variables of Nonautonomous Pneumatic Drive with Hydraulic Properties of Supply System
}

\author{
Yuri Pavlovich Kuznetsov ${ }^{1}$, Lev Anatolevich Zakharov ${ }^{1}$, Anatolii Alekseevich Mironov ${ }^{1}$, Vladimir Leonidovich \\ Khimich $^{1}$, Sergey Nikolaevich Khrunkov ${ }^{1}$
}

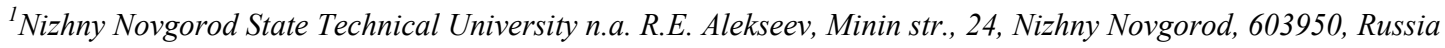

*Corresponding author E-mail: kuznetsov.yu.p@mail.ru

\begin{abstract}
This article discusses the issues of operability of nonautonomous pneumatic drive under workshop conditions. It is mentioned that in order to provide efficient operation and achievement of rated modes by nonautonomous pneumatic drive, it is required to coordinate its variables with variables of air supply system. On the basis of one-dimensional equation of adiabatic gas frictional flow in cylindrical pipe, the problem of determination of maximum achievable power by nonautonomous pneumatic drive at fixed hydraulic properties of supply system has been solved. The obtained dependences make it possible to calculate minimum allowed pipeline diameter of supply system providing preset maximum power of pneumatic drive. It is proved that the coefficient of total pressure conservation corresponding to maximum drive power is precisely determined by available pressure drop and does not depend on hydraulic properties of the system, and in order to achieve maximum drive power, strictly determined portion of available flow energy should be consumed for overcoming of the system hydraulic resistance not depending of reduced pipeline length.
\end{abstract}

Keywords: Air flow rate; Compressed air; Maximum power; Operation modes; Pneumatic drive.

\section{Introduction}

Nonautonomous pneumatic drive (hereinafter referred as the drive) operating from external source of compressed air is widely applied in mechanisms of ship and aircraft power plants as well as in machinery spindles and manual pneumatic tools $[1,2,3,4,5,6,7]$. Compressed air can be generated by individual compressor, air can be tapped from compressor stage of aircraft gas turbine engine or plant compressor station. Compressed air to the drive is supplied via pipeline system (hereinafter referred to as the system), herewith, the pressure before the drive could vary significantly from the pressure at input to the system as a consequence of hydraulic loss in pipeline and restriction losses. Without any special requirements to the system variables designer applies allowable pressure loss and determines respective hydraulic properties and design variables. In the case of certain special requirements, it is necessary to determine its hydraulic properties which would simultaneously provide the preset requirements and preset drive power, that is, it is required to coordinate variables of the drive and the system. For instance, while designing manual pneumatic grinding high-duty machines $[8,9,10,11,12,13,14,15]$, it is required to determine variables of supply sleeve which would provide simultaneously preset drive power of the machine and required flexibility of the supply sleeve. In extreme case it is required to determine minimum internal diameter of the supply sleeve which would provide the preset drive power. Let us consider solution of this problem with regard to pneumatic tool.

Principal flowchart of the system supplying compressed air from compressor to pneumatic machine is illustrated in Fig. 1. Compressed air from the compressor, passing the main pipeline 1 , the filter 2 , the pressure regulator 3 , the lubricator 4 , the blocking valve 5 and the sleeve 6 is supplied to the pneumatic machine 7 .
The equation of one-dimensional adiabatic gas frictional flow in cylindrical pipe has been described in [16]:

$\frac{1}{\lambda_{0}^{2}}-\frac{1}{\lambda_{1}^{2}}+\ln \frac{\lambda_{0}^{2}}{\lambda_{1}^{2}}=\chi=\frac{2 k}{k+1} \zeta \frac{l}{d^{\prime}}$

where $\mathrm{k}$ is the adiabatic index; $\zeta$ is the coefficient of pipe hydraulic resistance; $l, d$ are the pipe length and internal diameter, respectively; $\lambda=\frac{c}{a^{*}}$ is the dimensionless flow rate; $\mathrm{c}$ is the flow rate; $a^{*}$ is the critical flow rate.

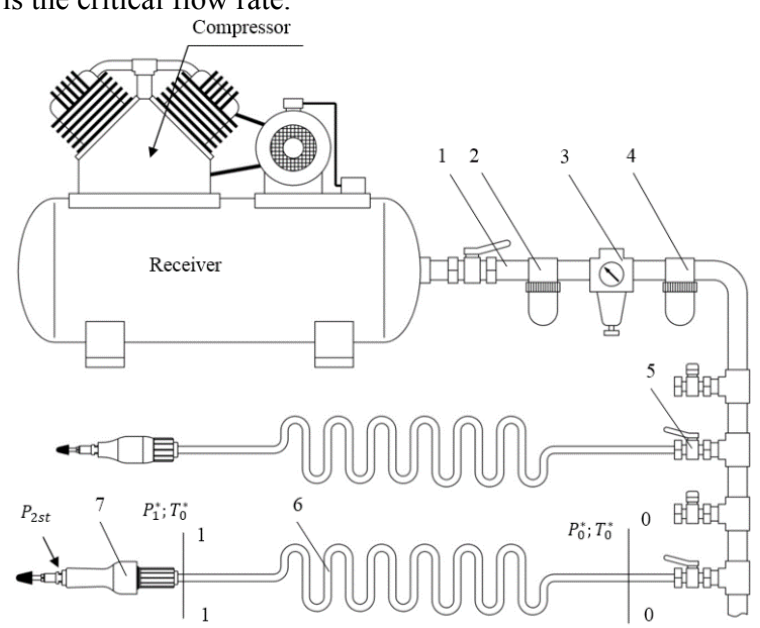

Fig. 1: Typical flowchart of compressed air supply to pneumatic machine: 1 - pipeline; 2 - filter-drier; 3 - pressure regulator; 4 - lubricator; 5 blocking valve; 6 - sleeve; 7 - pneumatic machine

In Eq. (1) the flow variables with index "0" are related to the input pipe cross section, and the variables with index "1" - to the output 
pipe cross section. The dimensionless variable $\chi$ is referred to as the pipe reduced length, it cumulatively expresses hydraulic properties of the supply system.

As illustrated in Fig. 1, the system is generally comprised of the cylindrical pipeline $(1,6)$ with various local restrictions: the filters (2), the pressure regulators (3), the lubricators (4), the blocking valves (5), various fittings, etc. For convenience of calculations it is allowed to express all local restrictions in terms of pipe equivalent length, its resistance equals to the sum of all local resistances: $\chi_{e q}=\frac{2 k}{k+1} \sum \zeta_{\text {loc }}$. Hereinafter, upon calculations of flows in the system the reduced length $\chi$ is the sum of the reduced length of cylindrical pipeline and the pipe reduced length substituting the local resistances.

\section{Analytical Solution}

Equation (1) is transcend, let us convert it in order to obtain analytical solution. Numerical calculations of variables of drive and supply system mentioned in [17] have revealed that practical range of variables $\chi$ and $\lambda$ is limited as follows: $\chi>20 ; \lambda<0.4$ With available pressure loss $\pi=\frac{P_{2 s t}}{P_{0}^{*}} \geq 0.05$ the ratio of flow rates in input and output cross sections is in the following range: $0.5 \leq$ $\frac{\lambda_{0}}{\lambda_{1}} \leq 0.95$. Respectively, $\ln \frac{\lambda_{0}^{2}}{\lambda_{1}^{2}}$ is in the range of: $-0.7 \leq \ln \frac{\lambda_{0}^{2}}{\lambda_{1}^{2}} \leq-$ 0.2 . Since $\chi$ is at least by an order of magnitude higher than $\ln \frac{\lambda_{0}^{2}}{\lambda_{1}^{2}}$, then it is possible to neglect the term $\ln \frac{\lambda_{0}^{2}}{\lambda_{1}^{2}}$ and Eq. (1) can be rewritten as follows:

$\frac{1}{\lambda_{0}^{2}}-\frac{1}{\lambda_{1}^{2}} \approx \chi$

In order to determine variations of deceleration pressure at the sleeve segment $0-1$ let us apply the coefficient of pressure conservation $\sigma=\frac{P_{1}^{*}}{P_{0}^{*}}=\frac{q\left(\lambda_{0}\right)}{q\left(\lambda_{1}\right)}$, where $q(\lambda)=\left(\frac{k+1}{2}\right)^{\frac{1}{k-1}} \lambda\left(1-\frac{k-1}{k+1} \lambda^{2}\right)^{\frac{1}{k-1}}$ is the gas dynamic function of the reduced flow rate.

In the range $\lambda<0.4$ the function of reduced flow rate $q(\lambda)$ varies in fact linearly as a function of $\lambda$, hence with error not higher than $3 \%$ it is possible to write as follows:

$q(\lambda) \approx\left(\frac{k+1}{2}\right)^{\frac{1}{k-1}} \lambda$

Then, with consideration for the aforementioned assumption:

$\sigma=\frac{P_{1}^{*}}{P_{0}^{*}} \approx \frac{\lambda_{0}}{\lambda_{1}}$

Let us multiply the left-side and right-side terms of Eq. (2) by $\lambda_{0}^{2}$ and, with consideration for Eq. (4), Eq. (2) will be rewritten as follows:

$1-\sigma^{2}=\lambda_{0}^{2} \chi$ or $\lambda_{0}=\chi^{-0,5}\left(1-\sigma^{2}\right)^{0,5}$.

Therefore, as a consequence of the applied transformations, the transcend Eq. (1) is substituted by the simple algebraic Eq. (5) directly interrelating the flow rate $\lambda_{0}$ with the coefficient of pressure conservation $\sigma$.

Let us write the equation of drive power:

$N=G H \eta_{e}$

where $G=\sqrt{\left\{\frac{k}{R}\left(\frac{2}{k+1}\right)^{\frac{k+1}{k-1}}\right\}} \frac{\pi d^{2}}{4} \frac{P_{0}^{*}}{\sqrt{T_{0}^{*}}} q\left(\lambda_{0}\right)$ is the gas mass flow;
$H=C_{p} T_{0}^{*}\left[1-\left(\frac{\pi}{\sigma}\right)^{\frac{k-1}{k}}\right]$ is the available heat drop; $\eta_{e}$ is the drive efficiency; $P_{0}^{*}, T_{0}^{*}$ are the pressure and the temperature of flow deceleration in the input sleeve cross section (0-0); $P_{2 s t}$ is the static pressure at output from engine; $\pi=\frac{P_{2 s t}}{P_{0}^{*}}$ is the available pressure drop; $C_{p}$ is the gas heat capacity; $\mathrm{R}$ is the gas constant, $d$ is the sleeve internal diameter; $\sigma$ is the coefficient of pressure conservation at the segment (0-1).

Under the predefined initial gas parameters variation of drive power is completely determined as follows: $\bar{N}=q\left(\lambda_{0}\right)[1-$ $\left.\left(\frac{\pi}{\sigma}\right)^{\frac{k-1}{k}}\right]$ which is referred to as reduced power. With increase in gas flow rate, the drive power increases at first to maximum and then decreases due to energy loss in the system.

Let us determine conditions of achievement of maximum drive power with consideration for energy loss in the system. Drive power can be written as follows:

$N=\left\{P_{0}^{*} \sqrt{T_{0}^{*} \frac{\pi d^{2}}{4}} C_{p} \chi^{-0,5} \eta_{e} \sqrt{\frac{k}{R} \frac{2}{k+1}}\right\} \bar{N}$.

Taking into account Eqs. (3) and (5) and reducing some parameters, we have:

$\mathrm{N}=\left\{P_{0}^{*} \sqrt{T_{0}^{*}} \frac{\pi d^{2}}{4} C_{p} \chi^{-0,5} \eta_{e} \sqrt{\frac{k}{R} \frac{2}{k+1}}\right\}\left(1-\sigma^{2}\right)^{0,5}\left[1-\left(\frac{\pi}{\sigma}\right)^{\frac{k-1}{k}}\right]$.

Let us introduce the following notation:

$A=P_{0}^{*} \sqrt{T_{0}^{*}} \frac{\pi d^{2}}{4} C_{p} \chi^{-0,5} \eta_{e} \sqrt{\frac{k}{R} \frac{2}{k+1}}$,

then $N=A\left(1-\sigma^{2}\right)^{0,5}\left[1-\left(\frac{\pi}{\sigma}\right)^{\frac{k-1}{k}}\right]$

Conditions of achievement of maximum drive power we determine from $\frac{\partial N}{\partial \sigma}=0$ :

$\frac{\partial \mathrm{N}}{\partial \sigma}=\frac{\partial}{\partial \sigma}\left\{A\left(1-\sigma^{2}\right)^{0,5}\left[1-\left(\frac{\pi}{\sigma}\right)^{\frac{k-1}{k}}\right]\right\}=0$.

The complex $A$ without variable $\sigma$ can be reduced. Therefore, parameters in the complex $A$ (including the pipe reduced length $\chi$ ) do not determine the conditions of achievement of (A)aximum power. After algebraic transformation Eq. (7) can be rewritten as follows:

$\frac{1}{k} \sigma_{N_{\max }}^{\frac{1-k}{k}}-\left(\frac{1-k}{k}\right) \sigma_{N_{\max }}^{\frac{1-3 k}{k}}=\pi^{\frac{1-k}{k}}$

where $\sigma_{N_{\max }}$ is the coefficient of pressure conservation corresponding to maximum achievable drive power with consideration for energy loss in the system.

Applying algebraic transformations, we obtain the following equation:

$\sigma_{N_{\max }}\left[\frac{1}{k}+\left(\frac{k-1}{k}\right) \sigma_{N_{\max }}-2\right]^{\frac{k}{1-k}}=\pi$

Let us analyze Eq. (8) aiming at obtaining its approximate analytical solution. Let us introduce the following notation:

$\mathrm{X}=\frac{1}{k} \sigma_{N_{\max }}^{\frac{1-k}{k}} ; \mathrm{Y}=-\left(\frac{1-k}{k}\right) \sigma_{N_{\max }} \frac{1-3 k}{k} ;$ 
$\mathrm{Z}=\frac{1}{k} \sigma_{N_{\max }}^{\frac{1-k}{k}}-\left(\frac{1-k}{k}\right) \sigma_{N_{\max }} \frac{1-3 k}{k}=\pi^{\frac{1-k}{k}}$

Plots of functions $\mathrm{X}, \mathrm{Y}$ and $\mathrm{Z}$ (at $\mathrm{k}=1.4$ ) are illustrated in Fig. 2. Their pattern demonstrates that $X$, the first term of Eq. (8), varies by an order of magnitude lower that the second term of this equation, $\mathrm{Y}$, hence, $\mathrm{X}$ can be substituted with constant.

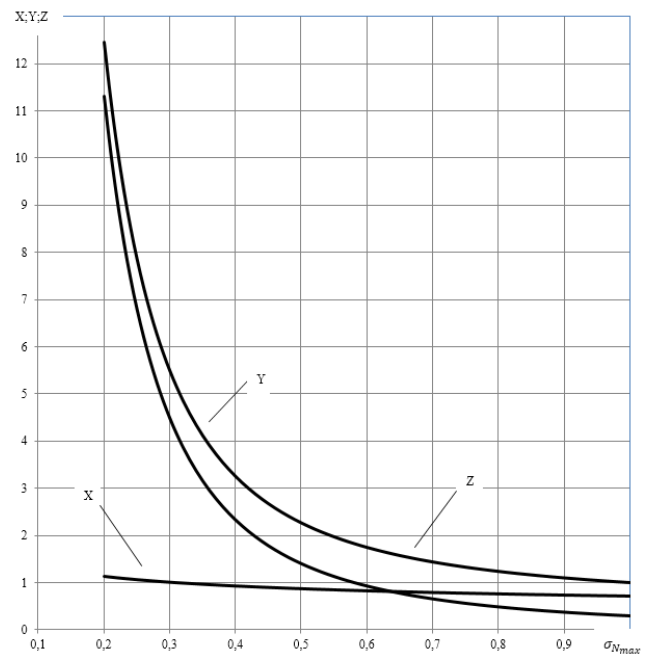

Fig. 2: $\mathrm{X}, \mathrm{Y}, \mathrm{Z}$ as a function of $\sigma_{N_{\max }}$

In order to retain boundary conditions, it is reasonable to set $\mathrm{X}=\frac{1}{k}$. Then, Eq. (8) can be written as follows: $\frac{1}{k}+\left(\frac{k-1}{k}\right) \sigma_{N_{\max }} \frac{1-3 k}{k}$ $=\pi^{\frac{1-k}{k}}$, and we obtain the equation of coefficient of pressure conservation $\sigma_{N_{\max }}$ corresponding to maximum achievable power $N_{\max }$ :

$\sigma_{N_{\max }}=\left\{\left(\frac{k}{k-1}\right)\left[\pi^{\frac{1-k}{k}}-\frac{1}{k}\right]\right\}^{\frac{k}{1-3 k}}$

It follows from Eq. (10) that $\sigma_{N_{\max }}$ is completely determined by available pressure drop $\pi=\frac{P_{2 s t}}{P_{0}^{*}}$ and does not depend on hydraulic properties of the system. The plots of $\pi=f\left(\sigma_{N_{\max }}\right)$ corresponding to Eqs. (9) and (10) are illustrated in Fig. 3.

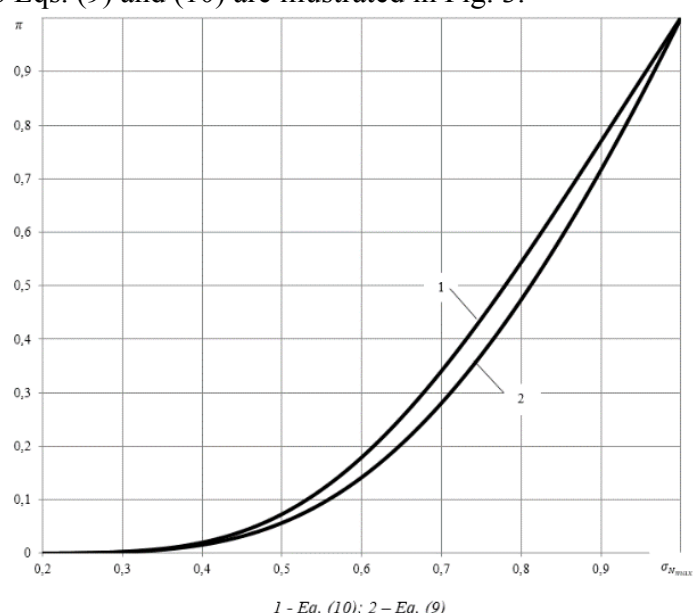

Fig. 3: Coefficient of pressure conservation as a function of available pressure drop

The difference in $\sigma_{N_{\max }}$ according to Eqs. (9) and (10) is in the range of $2 \ldots 7 \%$. The rate $\lambda_{0}$ corresponding to $N_{\max }$ is determined by Eq. (5) at $\sigma=\sigma_{N_{\max }}$ :

$\lambda_{0 N_{\max }}=\chi^{-0.5}\left(1-\sigma_{N_{\max }}^{2}\right)^{0,5}$
Hence, the mass flow rate corresponding to maximum achievable drive power is:

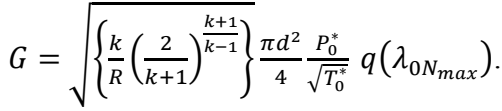

$N_{\max }$ at predefined $\pi$ and $\chi$ is determined by Eq. (6), and substituting $\sigma_{N_{\max }}$ and $\lambda_{0 N_{\max }}$ into it we obtain the following:

$N_{\max }=A \chi^{-0,5}\left(1-\sigma_{N_{\max }}{ }^{2}\right)^{0,5}\left[1-\left(\frac{\pi}{\sigma_{N_{\max }}}\right)^{\frac{k-1}{k}}\right]$

Therefore, the problem of maximum power $N_{\max }$ at known pipe reduced length $\chi$ is solved. In order to calculate minimum pipeline diameter of the system at predefined drive power, we apply Eq. (11). Using Eq. (1) let us express $d$ in terms of $\chi: d^{2}=$ $\left(\frac{2 k}{k+1} \zeta l\right)^{2} \chi^{-2}$ and substitute it into Eq. (11). Then, the equation for predefined power $N_{\text {def }}$ will be as follows:

$N_{\text {def }}=\left\{P_{0}^{*} \sqrt{T_{0}^{*} \frac{\pi}{4}} C_{p} \eta_{e}\left(\frac{2 k}{k+1} \zeta l\right)^{2} \sqrt{\frac{k}{R} \frac{2}{k+1}}\right\}\left(1-\sigma_{N_{\max }}^{2}\right)^{0,5}\left[1-\left(\frac{\pi}{\sigma_{N_{\max }}}\right)^{\frac{k-1}{k}}\right] \chi_{d_{\min }}{ }^{2,5}$.

Hence, it is possible to write as follows:

$\chi_{d_{\min }}=\left(\frac{N_{d e f}}{B}\right)^{-0,4}$,

where $\mathrm{B}=\left\{P_{0}^{*} \sqrt{T_{0}^{*}} \frac{\pi}{4} C_{p} \eta_{e}\left(\frac{2 k}{k+1} \zeta l\right)^{2} \sqrt{\frac{k}{R} \frac{2}{k+1}}\right\}\left(1-\sigma_{N_{\max }}{ }^{2}\right)^{0,5}\left[1-\left(\frac{\pi}{\sigma_{N_{\max }}}\right)^{\frac{k-1}{k}}\right]$.

Using Eqs. (12) and (13), we obtain the following equation:

$\left(\frac{l}{d}\right)_{d_{\min }}=\left(\frac{2 k}{k+1} \zeta\right)^{-1} \chi_{d_{\min }}$.

From Eq. (13) we determine $d_{\min }$ at predefined pipeline length $l$ of the system:

$d_{\min }=l\left(\frac{2 k}{k+1} \zeta\right) \chi_{d_{\min }}$

Therefore, the problem of maximum allowable pipeline diameter of the supply system is solved.

\section{Discussion}

The major conclusion of the performed analysis is that the coefficient of total pressure $\sigma_{N_{\max }}$ (Eq. (10)) does not depend on hydraulic properties of the system. The plot of reduced power $\bar{N}=f$ $(\sigma)$ illustrated in Fig. 4 demonstrates that at fixed $\pi$ the maximum power $\bar{N}$ for any $\chi$ is achieved at constant $\sigma_{N_{\max }}$. 


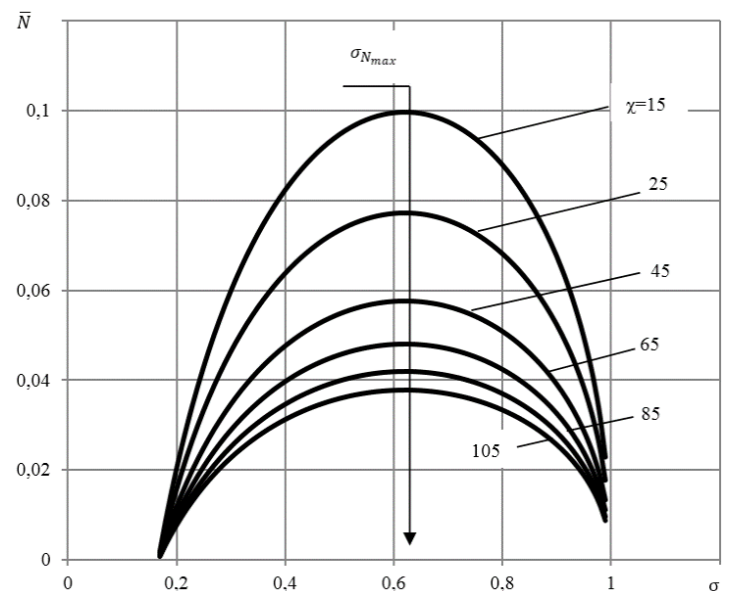

Fig. 4: Corrected power as a function of coefficient of pressure conservation and corrected pipeline length for available pressure drop $\pi=0.166$

Let us consider some consequences following from Eq. (10). It is obvious that increase in $\pi^{-1}=\frac{P_{0}^{*}}{P_{2 s t}}$ at predefined drive power leads to decrease in required gas flow rate, thus decreasing energy loss at hydraulic restrictions of the system. Less obvious is the influence of $\pi^{-1}$ on losses in the system during designing of drive for maximum achievable power. Energy loss in the system can be assessed by the ratio of reduced power $\bar{N}_{\text {max }}$ determined at preset $\chi$ to theoretical $\bar{N}_{\text {max }}$ determined at $\chi=0$ :

$\eta_{\text {SS }}=\frac{N_{\text {max }}}{N_{\text {theor }}}=\frac{q\left(\lambda_{0}\right) H_{N_{\max }}}{q\left(\lambda_{0}\right) H_{\text {avail }}}=\frac{1-\left(\frac{\pi}{\sigma_{N_{\max }}}\right)^{\frac{k-1}{k}}}{1-\pi^{\frac{k-1}{k}}}$.

The plots of $\sigma_{N_{\max }}=f\left(\pi^{-1}\right)$ and $\eta_{s s}=f\left(\pi^{-1}\right)$ illustrated in Fig. 5 demonstrate that $\eta_{s s}$ increases with pressure loss $\pi^{-1}$ despite that $\sigma_{N_{\max }}$ decreases with increase in $\pi^{-1}$. In workshop system it is assumed to apply standard pressure of $0.63 \mathrm{MPa}[18,19,20,21$, 22]. Analyzing the plots in Fig. 5 it is possible to conclude that the standard pressure of $0.63 \mathrm{MPa}$ in workshop system is quite reasonable, since the increase in $\pi^{-1}>6$ does not result in significant increase in $\eta_{s s}$.

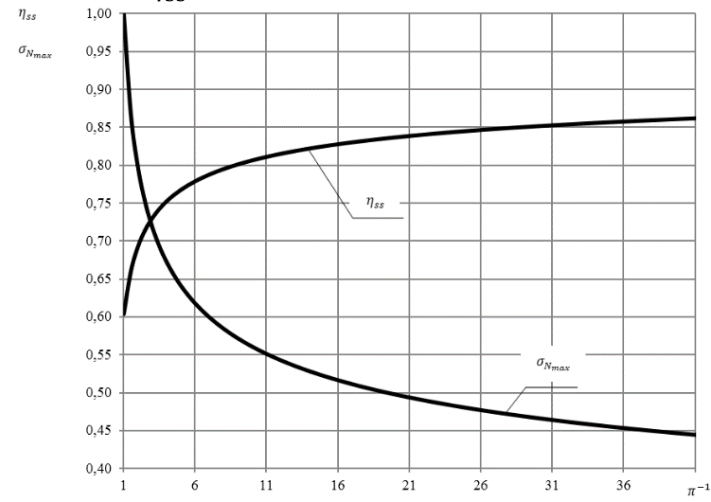

Fig. 5: Energy loss in $\eta_{s s}$ system and coefficient of pressure conservation $\sigma_{\bar{N}_{\text {max }}}$ as a function of available pressure drop $\pi$

It should be mentioned that at fixed $\pi$ the reduced heat drop $\bar{H}_{N_{\max }}=1-\left(\frac{\pi}{\sigma_{N_{\max }}}\right)^{\frac{k-1}{k}}=$ idem. Therefore, all maximum values of reduced power $\bar{N}_{\text {max }}$ determined at various $\chi$ will be located on straight line $\bar{N}_{\text {max }}=\left(\frac{k+1}{2}\right)^{\frac{1}{k-1}} \lambda_{0}\left[1-\left(\frac{\pi}{\sigma_{N_{\max }}}\right)^{\frac{k-1}{k}}\right]$ which is illustrated by the plots in Figs. 6 and 7.

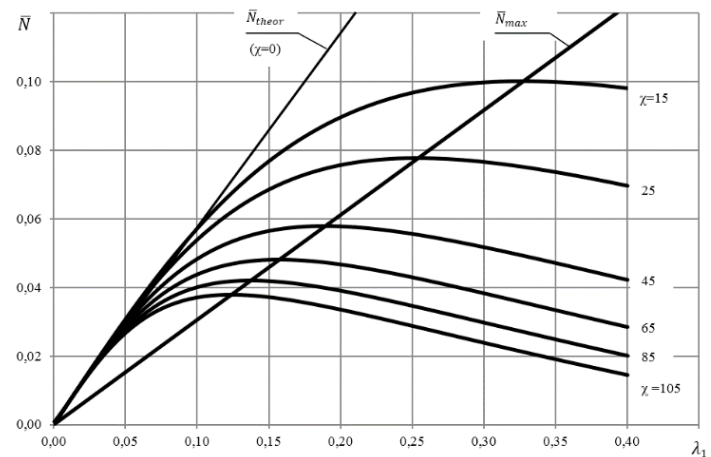

Fig. 6: Reduced power $\bar{N}$ as a function of dimensionless flow rate at input to nonautonomous drive $\lambda_{1}$ for $\pi=0.166$

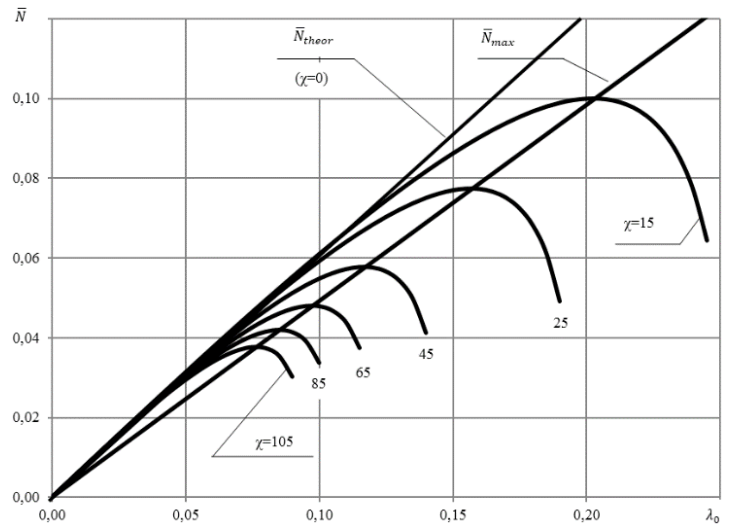

Fig. 7: Reduced power $\bar{N}$ as a function of dimensionless flow rate at input to supply sleeve of nonautonomous drive $\lambda_{0}$ for $\pi=0.166$

\section{Conclusions}

Let us summarize the main conclusions obtained during the performed analysis:

- the coefficient of total pressure conservation $\sigma_{N_{\max }}$ corresponding to maximum drive power is precisely determined by available pressure drop $\pi$ and does not depend on the system hydraulic properties;

- in order to achieve maximum drive power, strictly determined portion of available flow energy should be consumed for overcoming of the system hydraulic resistance not depending of reduced pipeline length $\chi$;

- while designing drive for maximum achievable power with increase in the available pressure drop $\pi^{-1}$ the variable $\eta_{s s}$ increases despite decrease in $\sigma_{N_{\max }}$

\section{References}

[1] Korenbaum VI, Tagiltsev AA, Gorovoi S.V, Kostiv AE, Shiryaev AD, Fershalov YuYa \& Maryutin VS (2017), A low-frequency power-type pressure-gradient receiver for oceanological investigations. Instruments and Experimental Techniques 60(5), 728-732.

[2] Kuznetsov YP, Khimich VL, Khrunkov SN \& Krainov AA (2016), Radial two-stage microturbine for pneumatic actuation. Russian Aeronautics 59(2), 283-286.

[3] Khimich VL, Chuvakov AB, Kikeyev VA, Khrunkov SN \& Kraynov AA (2016), Two-rimming radial turbine for drive of manual pneumatic grinders. International Journal of Applied Engineering Research 116(16), 8982-8986.

[4] Fershalov A, Fershalov Yu, Fershalov M et al (2016), Constructive and regime factors influence on turbine wheel characteristics with large rotation flow angle of blades. Polyarnaya mehanika 3, 976985.

[5] Fershalov AYu, Fershalov YuYa, Fershalov MYu, Tsygankova LP \& Korshunov VN (2015), Influence of constructive and regime factors on the characteristics nozzle apparatus with small angles output 
nozzle. Modern technologies and development of polytechnic education, 514-516.

[6] Fershalov AYu, Fershalov YuYa, Fershalov MYu, Tsygankova LP \& Korshunov VN (2015), Influence of design and operational factors on the efficiency of the microturbine stages with small angles of nozzle. Modern technologies and development of polytechnic education, 516-518.

[7] Fershalov AYu, Fershalov YuYa, Fershalov MYu \& Sazonov TV (2015), Results of the study rotor wheels supersonic microturbines with a large angle of rotation of the flow. Applied Mechanics and Materials 752-753, 884-889.

[8] Khimich VL, Chuvakov AB, Khrunkov SN \& Kraynov AA (2016), The influence of aerodynamic characteristics of the elements of the flow range of the radial two-row range of the radial two-row microturbine on its dynamic characteristics. International Journal of Applied Engineering Research 11(23), 11501-11509.

[9] Fershalov YuYa, Fershalov MYu, Fershalov AYu (2017), Energy efficiency of nozzles for axial microturbines. Procedia Engineering 206 499-504.

[10] Fershalov AY, Fershalov YuYa \& Tsigankova LP (2015), The degree of influence of constructive and regime factors on the characteristics turbine wheel steps shoulder who are more angles of rotation. RECENT ADVANCES in MATHEMATICS, Series "Mathematics and Computers in Science and Engineering Series", 130-133.

[11] Fershalov AYu, Fershalov MYu, Fershalov YuYa, Sazonov TV \& Ibragimov DI (2015), Research data of turbine nozzles of 5-9 degree outlet angles. Applied Mechanics and Materials 770, 547-550.

[12] Fershalov AYu, Fershalov YuYa, Fershalov MYu, Sazonov TV \& Ibragimov DI (2014), Analysis and optimization of efficiency rotor wheels microturbines. Applied Mechanics and Materials 635-637, 76-79.

[13] Fershalov MYu, Fershalov YuYa, Fershalov AYu, Sazonov TV \& Ibragimov DI (2014), Microturbines degree of reactivity. Applied Mechanics and Materials 635-637, 354-357.

[14] Fershalov YuYa (2012), Technique for physical simulation of gasodynamic processes in the turbomachine flow passages. Russian Aeronautics 55(4), 424-429.

[15] Fershalov MYu, Fershalov AYu \& Fershalov YuYa (2014), Calculation reactivity degree for axial low-account turbines with small emergence angles of nozzle devices. Advanced Materials Research 915-916, 341-344.

[16] Deich ME (1974), Tehnicheskaya gazodinamika [Engineering gas dynamics]. Moscow: Energiya.

[17] Kuznetsov YuP \& Kuznetsova TYu (2010), Soglasovanie parametrov neavtonomnogo pnevmaticheskogo privoda s gidravlicheskimi harakteristikami podvodyaschejj sistemy [Coordination of variables of nonautonomous pneumatic drive with hydraulic properties of supply system]. Trudy NGTU im. R.E. Alekseeva. T69, Energeticheskie ustanovki i teplotehnika 2, 86-94.

[18] Khimich VL, Chuvakov AB \& Khrunkov SN (2016), Maximum rotation frequency regulators of high-speed small-sized pneumatic actuators. International Journal of Applied Engineering Research 11(18), 9256-9260.

[19] Fershalov AYu, Fershalov MYu, Fershalov YuYa, Sazonov TV \& Ibragimov DI (2015), The design of the nozzle for the nozzle box microturbines. Applied Mechanics and Materials 789-790, 203-206.

[20] Fershalov YuYa \& Sazonov TV (2014), Experimental research of the nozzles. Advanced Materials Research 915-916, 345-348.

[21] Sazonov TV, Fershalov YuYa, Fershalov MYu, Fershalov AYu \& Ibragimov DI (2014), Experimental installation for the study of nozzles microturbines. Applied Mechanics and Materials 635-637, $155-158$

[22] Ibragimov D, Mochalov A \& Ilinskiy Yu (2017), Research Data of Microturbine Nozzles with Outlet Angles under 9 Degree (Conference Paper), Procedia Engineering 206, 493-498. 